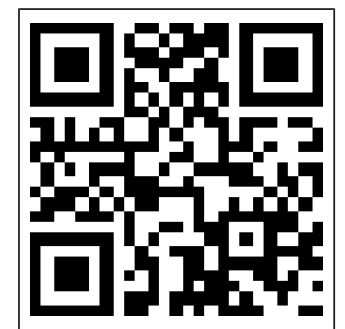

Scan to access more free content
- Additional materials are published online only. To view these files please visit the journal online (http://dx.doi.org/ 10.1136/gutjnl-2011-301824).

${ }^{1}$ Department of Medicine, Division of Digestive and Liver Disease, College of Physicians and Surgeons, Columbia University, New York, New York, USA

${ }^{2}$ Division of Comparative Medicine, Massachusetts Institute of Technology, Cambridge, Massachusetts, USA

${ }^{3}$ II. Medizinische Klinik, Klinikum rechts der Isar, Technische Universität München, Munich, Germany

\section{Correspondence to}

Dr Professor Timothy C Wang,

Chief, Division of Digestive and Liver Diseases, Silberberg

Professor of Medicine,

Department of Medicine and Irving Cancer Research Center Columbia University Medical Center, 1130 Street Nicholas Avenue, Room \#925, New York, NY 10032-3802, USA: tcw21@columbia.edu

Revised 4 January 2012 Accepted 11 January 2012 Published Online First 23 February 2012

\title{
Stromal cell-derived factor-1 overexpression induces gastric dysplasia through expansion of stromal myofibroblasts and epithelial progenitors
}

\author{
Wataru Shibata, ${ }^{1}$ Hiroshi Ariyama, ${ }^{1}$ Christoph Benedikt Westphalen, ${ }^{1}$ \\ Daniel L Worthley, ${ }^{1}$ Sureshkumar Muthupalani, ${ }^{2}$ Samuel Asfaha, ${ }^{1}$ \\ Zinaida Dubeykovskaya, ${ }^{1}$ Michael Quante, ${ }^{3}$ James G Fox, ${ }^{2}$ Timothy C Wang ${ }^{1}$
}

\section{ABSTRACT}

Objective Stromal cell-derived factor-1 (SDF-1/CXCL12) the main ligand for CXCR4, is overexpressed in human cancer. This study addressed the precise contribution of SDF-1 to gastric carcinogenesis.

Design SDF-1 transgenic mice were created and a Helicobacter-induced gastric cancer model was used in combination with $\mathrm{H} / \mathrm{K}$-ATPase-IL-1 $\beta$ mice. Gastric tissue was analysed by histopathology and cells isolated from the stomach were analysed by molecular biological methods.

Results Analysis of the H/K-ATPase/SDF-1 transgenic (SDF-Tg) mice showed that SDF-1 overexpression results in significant gastric epithelial hyperproliferation, mucous neck cell hyperplasia and spontaneous gastric dysplasia (wild-type mice 0/15 (0\%) vs SDF-Tg mice 4/14 (28.6\%), $p=0.042$, Fisher exact test) but has minimal effects on inflammation. SDF-Tg mice also showed a dramatic expansion of $\alpha$-smooth muscle actin-positive myofibroblasts and CXCR4-expressing gastric epithelial cells in the progenitor zone, both of which preceded the development of significant gastritis or dysplasia. Gremlin 1-expressing mesenchymal stem cells, the putative precursors of myofibroblasts, were also increased within the dysplastic stomachs of SDF-Tg mice and showed chemotaxis in response to SDF-1 stimulation. SDF-1 overexpression alone resulted in minimal recruitment of haematopoietic cells to the gastric mucosa, although macrophages were increased late in the disease. When SDF-Tg mice were crossed with H/K-ATPase-IL-1 $\beta$ mice or infected with Helicobacter felis, however, there were dramatic synergistic effects on recruitment of bone marrow-derived cells and progression to preneoplasia. Conclusion Activation of the SDF-1/CXCR4 axis can contribute to early stages of carcinogenesis primarily through recruitment of stromal cells and modulation of the progenitor niche.

\section{INTRODUCTION}

Gastric cancer is the second leading cause of cancer worldwide, and it is now well established that Helicobacter pylori-associated chronic inflammation plays a pivotal role in triggering the sequence from chronic gastritis to cancer. ${ }^{1}$ The active stromal remodelling that accompanies chronic inflammation is critical to the initiation and progression of cancer. $^{2}$ Thus, there has been increasing interest to identify the key chemokines and cytokines that

\section{Significance of this study}

What is already known on this subject?

- Stromal cell-derived factor-1 (SDF-1) and its cognate receptor CXCR4 mediate many biological functions such as embryonic development, organ homeostasis, angiogenesis and immune system modulation.

- Studies in murine cancer models have shown that SDF-1 produced by myofibroblasts recruits endothelial cells and contributes to tumorigenesis.

- SDF-1 mRNA and serum SDF-1 are upregulated in both mice and human gastric cancer and blocking SDF/CXCR4 reduces tumour growth and the development of ascites.

What are the new findings?

- Overexpression of SDF-1 in the stomach caused a significant increase in gastric epithelial cell proliferation, induced inflammation and promoted gastric tumour development, particularly in combination with inflammatory stimuli such as Helicobacter infection or interleukin $1 \beta$ (IL-1 $\beta$ ).

- SDF-1 recruited F4/80-positive macrophages and CD11b-positive myeloid cells and induced gastric epithelial proliferation partly through the activation of SDF/CXCR4 signalling and its downstream Erk/PI3kinase.

- SDF-1 overexpression recruited CXCR4-positive mesenchymal stem cells and the expansion of myofibroblasts in the gastric stem cell niche. This was associated with an increase in K19-positive epithelial cells.

- SDF-1 promoted gastric epithelial proliferation partly through CXCR4-positive gastric tissue stem/progenitor cells.

mobilise inflammatory and mesenchymal cells, leading to cancer. ${ }^{3}$

The chemokine stromal cell-derived factor-1 (SDF-1), also known as CXCL12, is a constitutively expressed and inducible chemokine that plays a fundamental role in embryonic development, organ homeostasis, angiogenesis and immune system modulation. SDF-1 binds to and initiates 


\section{Significance of this study}

How might it impact on clinical practice in the foreseeable future?

- SDF-1 drives cancer through cytokine-related recruitment of macrophages and chemotaxis of bone marrow-derived mesenchymal stem cells/myofibroblasts.

- In addition, the SDF-1/CXCR4 pathway plays a key role in the development of gastric carcinogenesis through direct modulation of CXCR4-positive stem/progenitor epithelial cells. SDF/ CXCR4 signalling is an attractive target for the prevention and treatment of gastric cancer.

signalling through two G-protein-coupled receptors, CXCR4 and CXCR7. In the bone marrow (BM), SDF-1 plays a critical role in the initial localisation, retention and support of CXCR4 haematopoietic stem cells (HSCs). ${ }^{4}$ SDF-1 is highly expressed in $\mathrm{BM}$ stromal cells and contributes to the BM niche. Gene knockout of either SDF-1 or CXCR4 results in impaired haematopoiesis and embryonic lethality, ${ }^{5-7}$ and inactivation of the SDF/CXCR4 signalling pathway promotes the mobilisation of HSCs into peripheral blood ${ }^{8}$ or mobilisation of neutrophils. ${ }^{9}$ Taken together, there is strong evidence that SDF-1/CXCR4 interactions modulate the engraftment, retention and release of haematopoietic cells from the BM.

It is not known whether SDF-1 also promotes the mobilisation and recruitment of haematopoietic cells into peripheral tissues. Indeed, SDF-1 is often upregulated in damaged tissues as a result of hypoxia or cellular apoptosis and increased SDF-1 plasma levels correlate with mobilisation of proangiogenic BM cells. ${ }^{10}$ The cognate receptor for SDF-1, CXCR4, is expressed on most BM-derived haematopoietic cells, including HSCs, endothelial precursor cells, immature myeloid cells, macrophages and lymphocytes ${ }^{11}$ and, through activation of phosphoinositide 3kinase, can regulate chemotaxis. While release of local SDF-1, such as following ischaemia, can mobilise haematopoietic cells, ${ }^{12}$ the recruitment of inflammatory cells is usually limited in the absence of injury, suggesting that SDF-1 is not sufficient for BM-derived cell recruitment. ${ }^{13} 14$

SDF-1 is one of the major chemokines consistently overexpressed in most solid tumours, ${ }^{15}{ }^{16}$ where it contributes to carcinogenesis as an autocrine growth factor as well as promoting angiogenesis and the recruitment of BM cells to the tumour microenvironment. ${ }^{17-19}$ While the CXCR4 receptor is primarily localised to the cancer cells, ${ }^{11}{ }^{20}$ the major source of the SDF-1 ligand in solid tumours appears to be stromal cells, particularly cancer-associated fibroblasts (CAFs). ${ }^{17}$ CAFs exhibit properties of myofibroblasts, including expression of $\alpha$-smooth muscle actin ( $\alpha \mathrm{SMA})$, and promote the growth of tumours through their ability to secrete SDF-1. ${ }^{17}$ Recent studies indicate that BM-derived mesenchymal stem cells (MSCs) represent one potential source for CAFs, and MSCs recruited into the cancer microenvironment are able to differentiate into CAF-like myofibroblasts. ${ }^{21-23}$ SDF-1 secreted by CAFs is important for both the migration and survival of MSCs in vitro. ${ }^{19}$ The overexpression of interleukin $1 \beta$ (IL-1 $\beta$ ) as well as chronic gastric Helicobacter infection both increase SDF-1 expression in the gastric mucosa. Furthermore, the recruitment of MSCs and CAFs, mediated by both SDF-1 and transforming growth factor (TGF) $\beta$, resulted in hyperproliferation of gastric epithelial cells. ${ }^{19}$ Thus, SDF-1 released by CAFs potentially recruits both
MSCs and CAFs, constituting the functional mesenchymal niche from the BM to sites of chronic injury. This recruited mesenchymal niche is likely to promote local stromal remodelling as well as cancer development. ${ }^{10} 19{ }^{24}$ Nevertheless, the full contribution of SDF-1 in gastric carcinogenesis is still unknown. In order to better define the precise role of SDF-1 in gastric carcinogenesis, we generated mice overexpressing SDF-1 in gastric parietal cells and examined its relevance to epithelial and stromal events in cancer.

\section{MATERIALS AND METHODS}

All animal studies were approved by the Institutional Animal Care and Use Committee at Columbia University. SDF transgenic mice were generated as described in the online supplement. CXCR4-EGFP mice were provided by Richard J Miller (Northwestern University Medical School, Chicago, Illinois, USA). Details of the mice used in this study, all protocols for bacterial culture, chronic $H$ felis infection model, histological evaluation, immunohistochemical studies, ELISA, real-time qRT-PCR assay of $H$ felis infection in mouse stomachs, proinflammatory CC chemokines and cytokines are given in the online supplement.

\section{RESULTS}

\section{Gastric overexpression of SDF-1 results in development of spontaneous gastric cancer}

We generated $\mathrm{H} / \mathrm{K}-\mathrm{ATPase} / \mathrm{hSDF}-1 \alpha$ transgenic mice (SDF-Tg) that expressed murine SDF-1 $\alpha$ specifically in gastric parietal cells (supplementary figure 1A). Two independent lines (lines 3 and 6) of SDF-Tg mice were identified by ELISA of gastric mucosa for SDF-1 or v5 tag (supplementary figure 1B-E). We confirmed that the murine Met-SDF-1V5x6His protein derived from the construct was biologically active and could induce lymphocyte chemotaxis in a dose-dependent manner, and the tagged SDF-1 protein bound surface proteoglycans with only slightly less efficiency than commercial recombinant SDF-1 (PeproTech Inc., Rocky Hill, NJ, USA.) lacking initial Met (data not shown). We backcrossed SDF-Tg mice to C57B6/J mice at least six times prior to further studies.

SDF-Tg mice exhibited markedly elevated expression of SDF-1 mRNA in the gastric mucosa (supplementary figure 1E), which was greater than the expression level observed in the BM. Interestingly, SDF-Tg mice aged between 3 and 12 months showed minimal inflammation in the stomach but nevertheless exhibited gastric hyperplasia and metaplasia. Young SDF-Tg mice (2-6 months) showed only a slight increase in the number of inflammatory cells determined by immunohistochemistry and FACS (supplementary figure 2A,B). By 18 months of age, however, the SDF-Tg mice exhibited dysplasia with marked cystic dilation of glands in corpus and antral tumours (figure 1). Furthermore, we observed a significant increase in chronic inflammatory cells at these later time points. While not evident in younger mice (not shown), there was a significant increase in the number of F4/80-positive cells and myeloperoxidase activity in older SDF-Tg mice (>12 months) compared with wild-type (WT) mice (supplementary figure 2C).

These results suggest that SDF-1 is able to induce gastric neoplasia despite minimal early effects on the recruitment of chronic inflammatory cells. In particular, the findings implied that, while SDF-1 is clearly able to recruit and retain CXCR4positive haematopoietic cells within the $\mathrm{BM}^{25}$ overexpression of SDF-1 in the stomach alone is not sufficient to induce the recruitment of haematopoietic cells to the stomach. We further tested this concept by transplantation of SDF-Tg and WT mice 
Figure 1 SDF-Tg mice developed spontaneous gastric dysplasia. Representative macroscopic and histological micrographs from SDF-Tg mice at 18 months (original magnifications $\times 100)$.

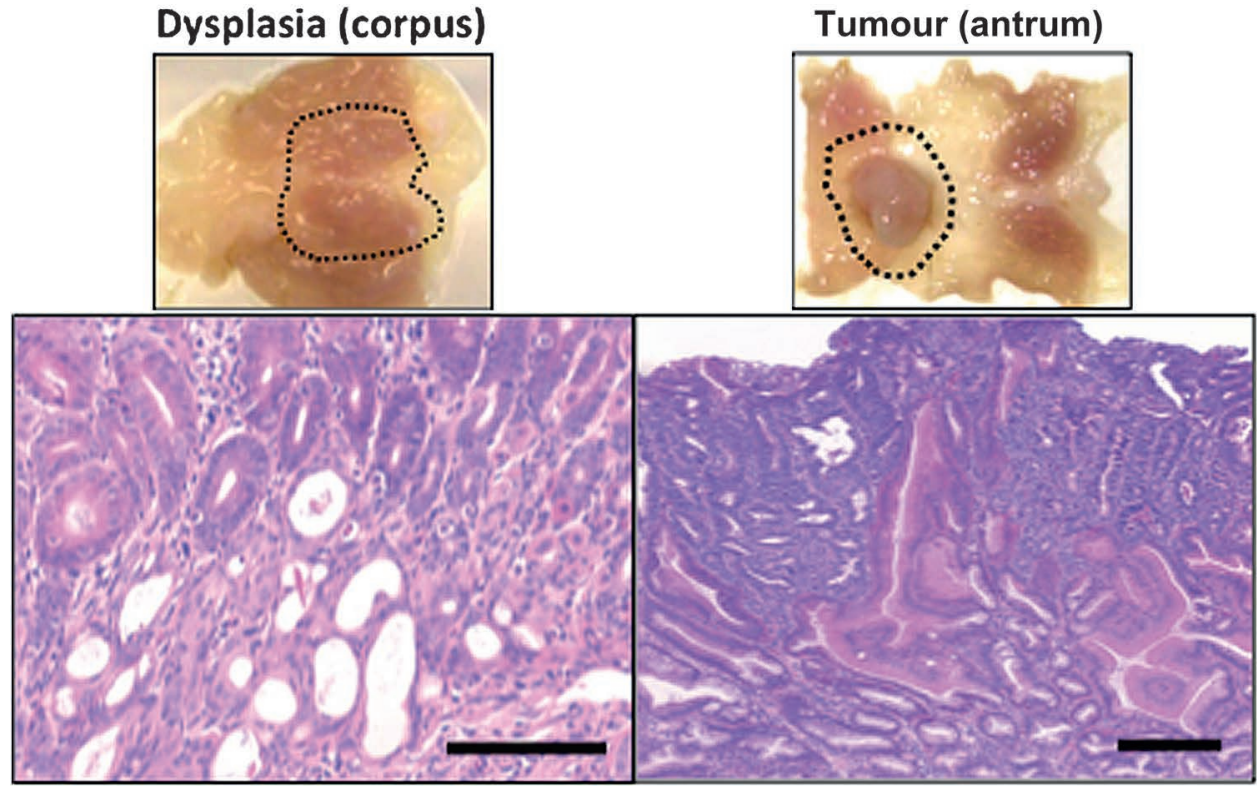

with GFP-labelled BM and followed the mice for up to 1 year. SDF-Tg transgenic mice showed a slight increase in GFP-labelled haematopoietic cells compared with WT mice at 1 year (data not shown), indicating that overexpression of SDF-1 alone has a weak and/or indirect effect on the recruitment of haematopoietic cells to the stomach. Nevertheless, the findings suggest that SDF-1 is able to drive epithelial proliferation independent of effects on haematopoietic cells. There were no histological alterations in other organs examined including intestine, liver, lung, and kidney in SDF-Tg mice (data not shown).

\section{SDF-1 overexpression accelerates inflammation-induced gastric tumorigenesis}

Given the absence of robust inflammation and the overall low incidence of gastric neoplastic lesions in SDF-Tg mice, we postulated that the induction of chronic inflammation in these animals might further accelerate the development of neoplasia. Thus, we employed $H$ felis infection in SDF-Tg mice in order to analyse the impact of SDF-1 overexpression in an established model of inflammation-associated gastric carcinogenesis. ${ }^{26}$ The mice were all of the same mixed genetic background (C57BL/6× CBA), and examined at time points up to 18 months after $H$ felis infection (MPI). Every histopathological parameter tended to be more severe in $H$ felis-infected SDF-Tg mice compared with $H$ felis-infected WT mice up to $12 \mathrm{MPI}$. At time points between 15 and $18 \mathrm{MPI}$ there were even more prominent histopathological changes, with significantly greater degrees of pseudopyloric metaplasia, oxyntic atrophy and foveolar hyperplasia in $H$ felis-infected SDF-Tg (figure 2A,B). Interestingly, only the $H$ felis-infected SDF-Tg mice exhibited gastric dysplasia and mucous metaplasia at 15-18 MPI, while no $H$ felis-infected WT mice developed severe dysplasia. Additionally, SDF-Tg mice infected with $H$ felis showed increased gastric expression of IL-1 $\beta$, heparin-binding epidermal growth factor and amphiregulin (figure 2C) and increased serum levels of IL-6 compared with WT mice infected with $H$ felis (figure $2 \mathrm{D}$ ).

The findings regarding IL- $1 \beta$ were particularly interesting, given previous studies in humans that had pointed to an important role for the IL-1 $\beta$ gene locus in gastric cancer susceptibility. ${ }^{27}$ Since gastric specific overexpression of IL- $1 \beta$ in transgenic mice was shown to be sufficient for the recruitment of myeloid cells to the stomach and the induction of gastric cancer, ${ }^{18}$ we crossed H/K-ATPase-IL-1 $\beta$ mice with SDF-Tg mice in order to determine the impact on carcinogenesis. At very early time points (eg, $1-1.5$ months) we found that SDF-1/IL-1 $\beta$ double transgenic mice showed significantly increased inflammatory cell infiltration, severe gastric atrophy and intestinal metaplasia in stomach compared with IL-1 $\beta$ single transgenic mice (supplementary figure 3A,B). The levels of IL-6 in gastric tissue were also significantly higher in SDF-1/IL-1 $\beta$ double transgenic mice compared with IL- $1 \beta$ single transgenic mice (supplementary figure $3 \mathrm{C}$ ). In combination with either $H$ felis infection or IL-1 $\beta$ overexpression, the number of F4/80 and CD11b-positive cells was significantly increased in SDF-Tg mice compared with their respective controls (figure $3 \mathrm{~A}, \mathrm{~B}$ ). These data suggest that, although SDF-1 alone is not sufficient to strongly promote chronic gastritis, it synergises with Helicobacter infection or IL-1 $\beta$ overexpression in the recruitment of myeloid cells and the induction of atrophic gastritis and gastric preneoplasia.

\section{SDF-1 extends survival and promotes the function of myeloid cells in vitro}

Given that SDF-1 synergised with IL-1 $\beta$ in the induction of gastritis, we wondered whether SDF-1 overexpression could increase the abundance of macrophages and the development of dysplasia through an effect on macrophage survival, as previously suggested. ${ }^{7}$ We isolated primary peritoneal macrophages using $4 \%$ thioglycolate and then stimulated the macrophages with either recombinant SDF-1 (rSDF-1) or gastric extract from SDF-Tg mice or WT mice. After $48 \mathrm{~h}$ of serum-free incubation, primary macrophages showed a significant increase in survival after administration with either $\mathrm{SSDF}-1$ or gastric extract from SDF-Tg mice (supplementary figure 4A). We also analysed the effect of SDF-1 on cytokine production in primary macrophages. There was no IL-6 production after stimulation with rSDF-1 alone; however, rSDF-1 administration showed a significant synergistic effect on the production of IL-6 from primary macrophages co-stimulated with lipopolysaccharide (supplementary figure $4 \mathrm{~B}$ ). We found that the increase in cytokine production was also associated with NF- $\kappa B$ signalling, confirmed by experiments using NF-KB inhibitor, MG132 (data not shown). Taken together, these results suggest that, apart from 
A
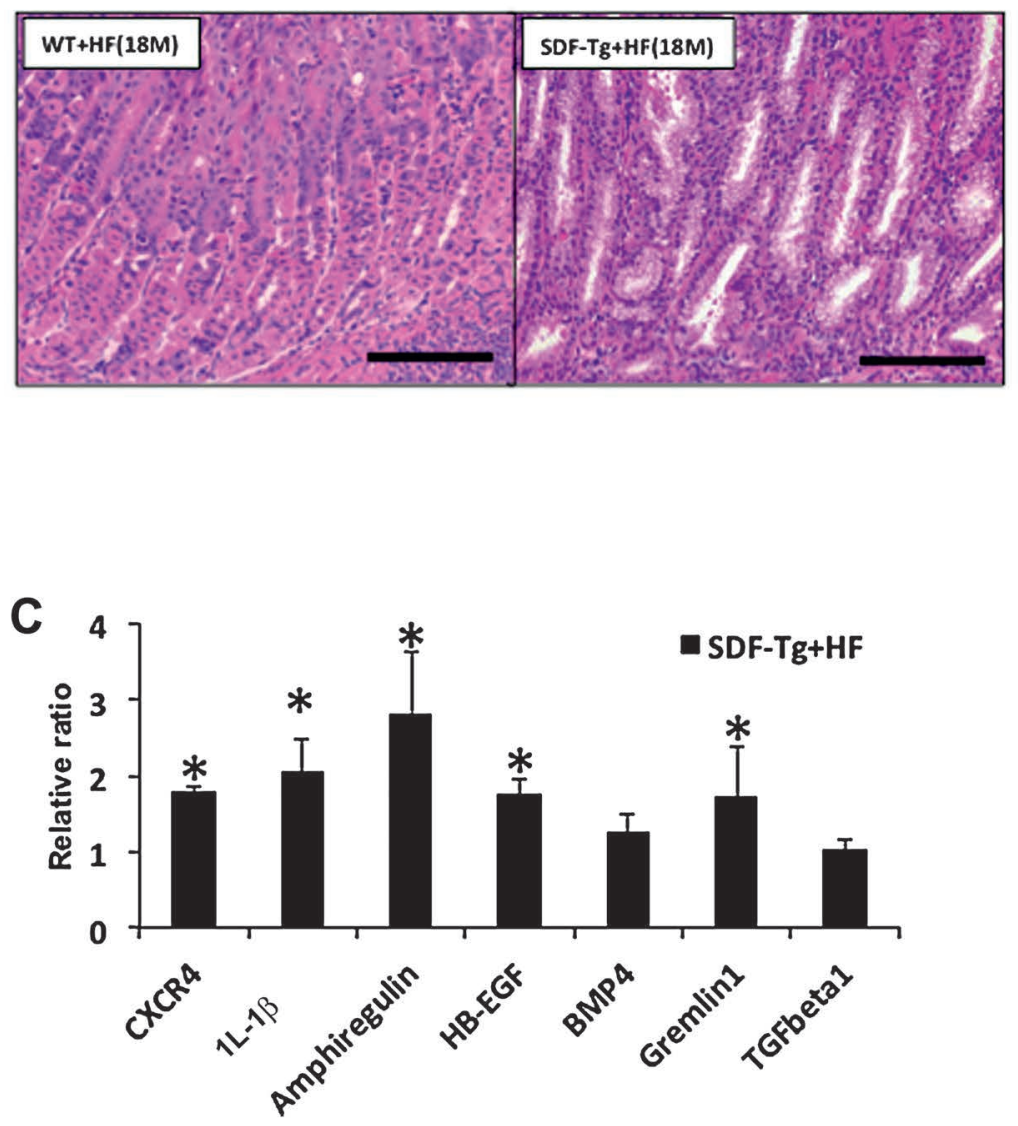

B (15-18 M/HF)

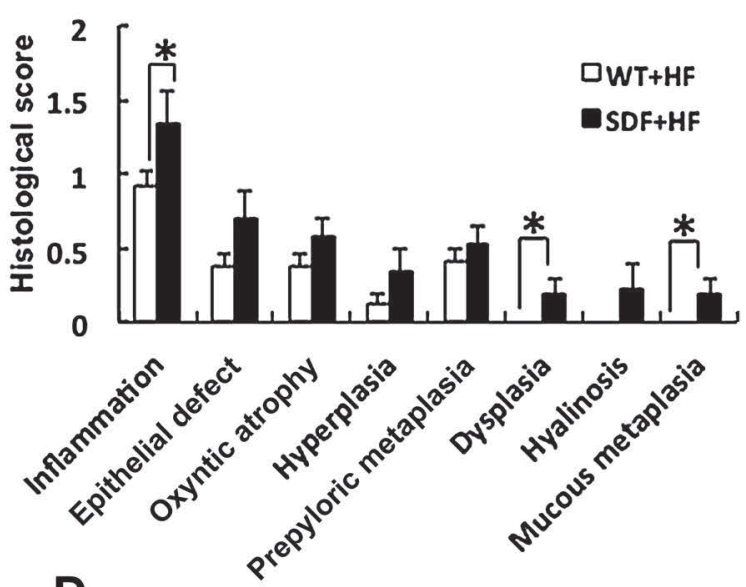

D

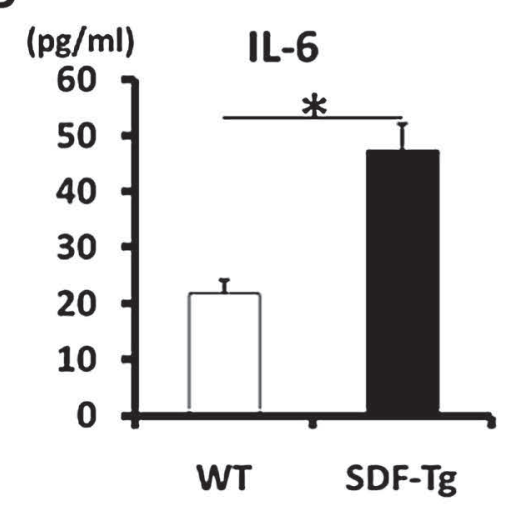

Figure 2 Overexpression of stromal cell-derived factor-1 (SDF-1) accelerates the development of gastric inflammation and dysplasia in the setting of Helicobacter felis infection. (A) Representative stomach sections. (B) Pathological scores from SDF-Tg mice and WT mice infected with $H$ felis for $15-18$ months ( $n=5$ per group). (C) qRT-PCR of gastric mucosa for the indicated genes. Gene expression was normalised to $10^{4}$ copies of GAPDH levels and the expression of each gene relative to WT mice is shown ( $=5$ per group). (D) Serum interleukin 6 (IL-6) levels from WT and SDF-Tg mice after $15 \mathrm{MPI}$ were measured by ELISA ( $n=5$ per group). Data shown are mean \pm SE. ${ }^{*} p<0.05$.

its role as a weak chemoattractant for myeloid cells, SDF-1 directly contributes to the survival and function of myeloid cells.

\section{SDF-1 induces epithelial proliferation and hyperplasia in part through CXCR4}

SDF-1 overexpression increases gastric epithelial proliferation. Immunostaining for Ki67 was increased in both the corpus and antrum of SDF-Tg mice compared with WT mice, irrespective of $H$ felis infection (figure 4A and supplementary figure 5A). Compared with WT mice, the increase in gastric proliferation in SDF-Tg mice led over time to gastric hyperplasia, particularly in the gastric pit and mucous neck regions. The number of K19positive cells, previously suggested to be gastric pit and progenitor cells, ${ }^{28}$ was significantly increased in SDF-Tg mice compared with WT mice (supplementary figure 5B). The number of parietal cells, however, was not significantly different in SDF-Tg mice and WT mice, suggesting that overexpression of SDF-1 somehow promoted the differentiation of the gastric epithelial stem cells towards the K19-expressing pit cell lineage, but not the glandular lineage. ${ }^{29}$

In order to confirm that the CXCR4 receptor mediated the proliferative response to SDF-1, we administered the CXCR4 antagonist AMD3100 to a cohort of SDF-Tg mice and WT controls. AMD3100 was administered to mice for 2 weeks. The mice were then killed and gastric epithelial proliferation assessed by Ki-67 immunostaining. AMD3100 significantly reduced the gastric epithelial proliferation index of the SDF-Tg mice to a level approaching that of untreated WT mice (figure 4B), consistent with a CXCR4-dependent pathway. Moreover, phosphorylatedAkt (p-Akt) and -Erk (p-Erk) could be detected in the bottom third of the antral glands where CXCR4-expressing cells reside, and $\mathrm{p}$-Akt and p-Erk positive cells were significantly increased both in the antrum and the corpus of SDF-Tg mice (figure 4C and supplementary figure 6). The increase in p-Akt or p-Erk was abrogated by the administration of AMD3100 (figure 4C and supplementary figure 6). Activation of CXCR4 by SDF-1 $\alpha$ has previously been shown to result in the activation of ERK and PIBK/Akt pathways, and downstream effectors of these pathways are reported to include IL- $6 .{ }^{30}$ Taken together, these data suggest that SDF-1 $\alpha$ stimulates gastric epithelial proliferation through Erk and PI3K/Akt-dependent mechanisms.

We have recently shown that the trefoil factor 2 (TFF2) functions as a partial antagonist to the CXCR4 receptor, with inhibitory effects on chemotaxis in response to SDF-1 through competitive inhibition of CXCR $4 .{ }^{31}$ We therefore crossed SDFTg mice with TFF2 null mice to test whether endogenous TFF2 might dampen the SDF-1-dependent proliferation and inflammation. SDF/TFF2-/- mice at 12 months of age showed increased proliferation and a dramatic increase in histopathological scores for gastric inflammation, oxyntic atrophy, 
Figure 3 SDF-Tg mice showed increase in F4/80- and CD11b-positive macrophages in stomach with Helicobacter felis infection or interleukin $1 \beta$ (IL-1 $\beta$ ) overexpression. (A) Number of F4/80-positive cells per high power fields in WT and SDF-Tg mice determined by counting positive cells per high power fields $(n=5$ per group, 15-month-old mice). (B) F4/80- or CD11b-positive cells were increased in SDF/IL-1 $\beta$-Tg mice; representative micrographs from stained paraffin sections from IL-1 $\beta$ and SDF/LL-1 $\beta$ mice $(n=5$ per group). Original magnification $\times 100$. Data shown are mean \pm SE. ${ }^{*} \mathrm{p}<0.05$ in each comparison indicated.
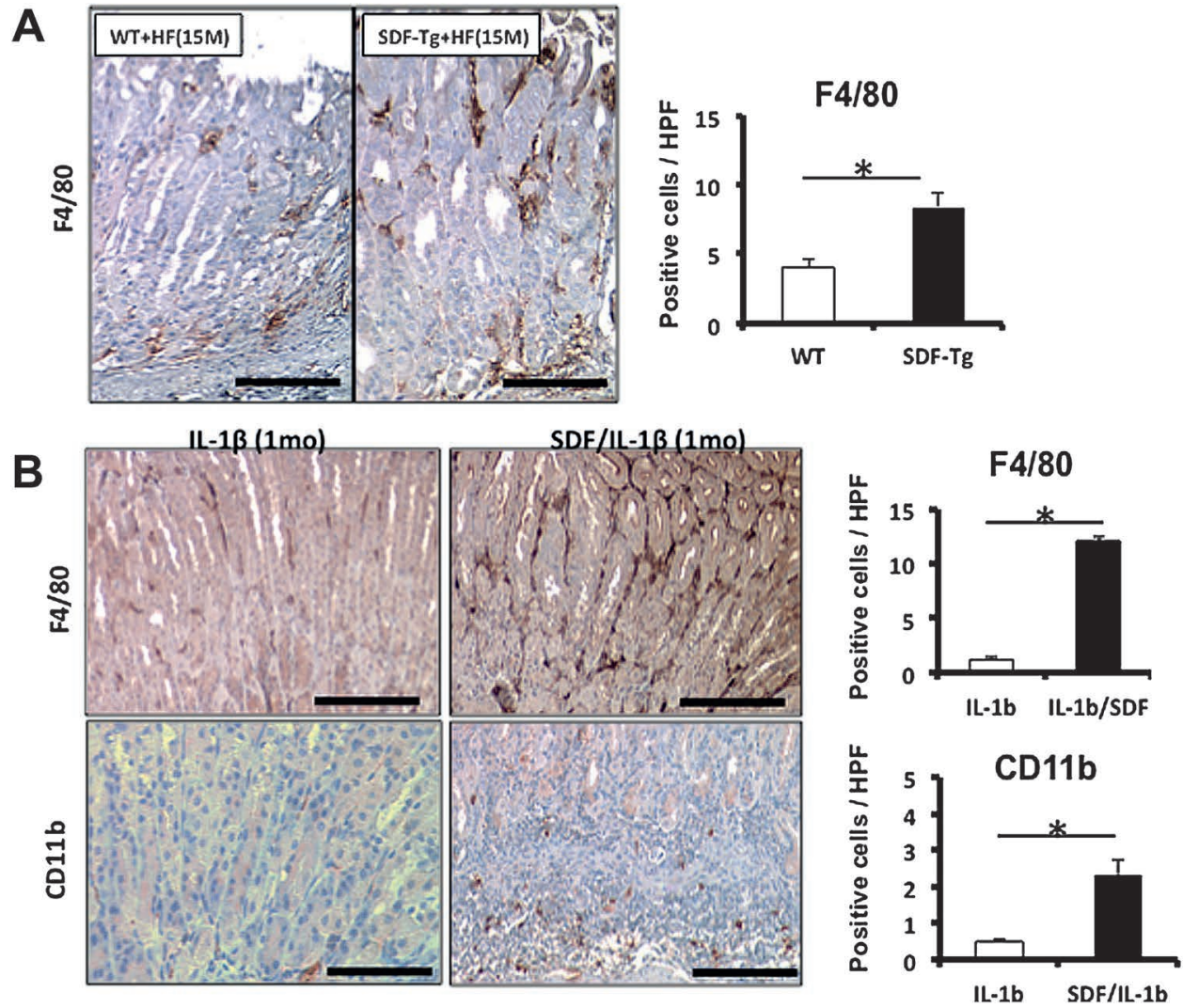

hyperplasia and metaplasia compared with SDF-Tg alone (supplementary figure 7A,B). In addition, we noted that SDF-1/ TFF2-/- mice showed markedly increased epithelial proliferation compared with age-matched SDF-1 transgenic mice as assessed by Ki67 immunohistochemistry (supplementary figure 7C,D). These results suggest that the development of gastric preneoplasia in SDF-Tg mice involves activation of the SDF/CXCR4 signalling pathway.

To determine whether overexpression of SDF-1 directly induced gastric epithelial proliferation, we identified potential SDF-1 target cells expressing the CXCR4 receptor in the stomach mucosa using CXCR4-EGFP BAC transgenic mice (supplementary figure 8A). ${ }^{32}$ In the corpus these CXCR4-EGFP $(+)$ cells were limited to the gastric isthmus, the known progenitor zone of the oxyntic mucosa. Furthermore, CXCR4EGFP $(+)$ cells were increased when CXCR4-EGFP mice were crossed with SDF-Tg mice compared with WT-CXCR4-EGFP mice (supplementary figure $8 \mathrm{~B}$ ). In the gastric antrum, however, the bottom third of the glands showed strong GFP-positive signals that appeared to overlap with the known location of antral stem and progenitor cells (supplementary figure 8C). ${ }^{33}$ Taken together, these data are consistent with a model in which overexpression of SDF-1 in the stomach could promote gastric epithelial cell proliferation directly, at least in part, through activation of CXCR4-expressing gastric progenitor cells.

\section{SDF-1 induces expansion of stromal myofibroblasts through recruitment of CXCR4-expressing MSCs}

While there were some gastric epithelial cells in the corpus that were positive for CXCR4-EGFP, there were clearly multiple stromal cells in the region of the gastric progenitor zone (isthmus) that were also positive for CXCR4-EGFP (supplementary figure 8A). Many of these had the appearance of stromal fibroblasts or myofibroblasts. Recent studies have suggested that $\alpha \mathrm{SMA}$-positive myofibroblasts contribute to the gastric stem cell niche, ${ }^{34}$ and that expansion of these cells contributes to the development of gastric neoplasia. ${ }^{19}$ Consequently, we examined the effects of SDF-1 overexpression on of aSMA-positive myofibroblasts by crossing SDF-Tg mice with aSMA-RFP (red fluorescent protein) reporter mice ${ }^{19}$ to generate SDF-Tg/aSMA-RFP double transgenic mice. We found that aSMA-positive cells were significantly increased in SDF-Tg mice compared with WT mice as early as the age of 4 months (figure $5 \mathrm{~A}$ ). Similar results were obtained using immunostaining for aSMA in both SDF-Tg mice and SDF-Tg/IL-1 $\beta$ double transgenic mice (data not shown). Taken together, these findings suggest that overexpression of SDF-1 is able to induce the early expansion of aSMA-positive myofibroblasts within the isthmus region of the gastric corpus.

Given the expansion of stromal cells resulting from the overexpression of SDF-1 in the stomach, we analysed mRNA expression of CXCR4 and Gremlin 1 (Grem1). Gremlin 1, a bone morphogenetic protein antagonist, is a recently identified marker of MSCs and is highly expressed by cancer-associated stromal cells. ${ }^{19} 35$ While SDF-Tg mice alone showed a small but significant increase in Grem 1 mRNA expression, SDF-Tg mice infected with $H$ felis showed significant upregulation of both Grem1 and CXCR4 mRNA expression in the stomach (figure 2C). These findings are consistent with SDF-1-dependent expansion and recruitment of MSCs to the stomach as recently described. ${ }^{19}$ A subset of MSCs has been shown to strongly express CXCR4 capable of promoting migration to $\mathrm{BM}^{36}$ We confirmed Grem 1 expression on CXCR4 positive stromal cells, by Grem1 immunostaining of our CXCR4-EGFP mouse (supplementary figure 9B) and that these Grem 1 positive cells were BMderived (figure $5 \mathrm{~B}$ ). We also showed a positive correlation of mRNA expression of CXCR4 (Spearman rank correlation test, $\rho=0.9, p=0.002$ ). mRNA expression of Grem 1 was upregulated 
A
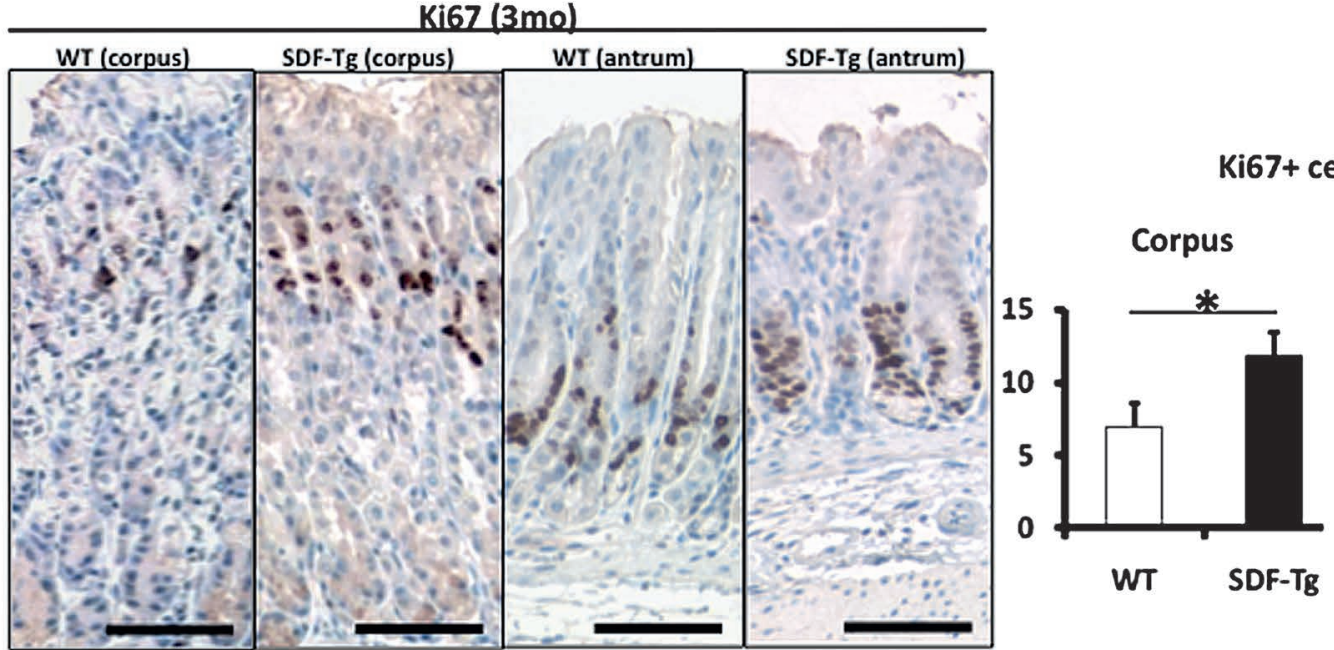

Ki67+ cells / gland
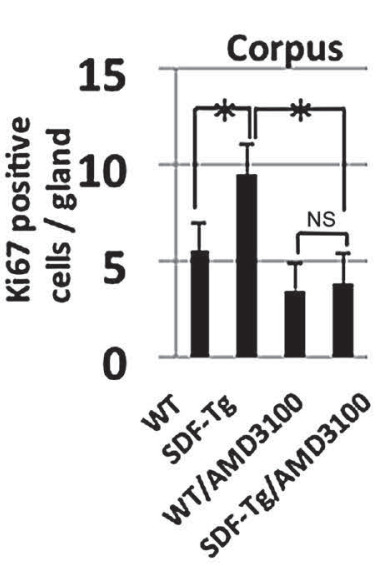

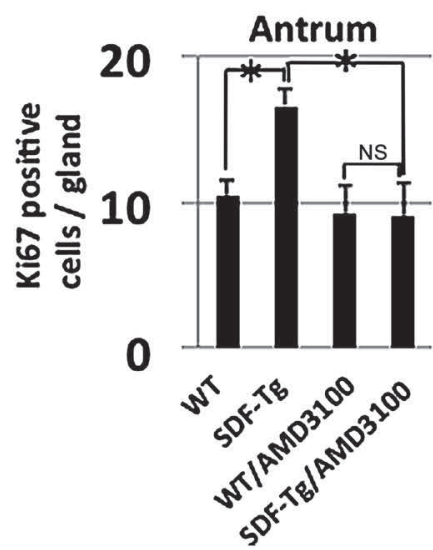

C

Corpus

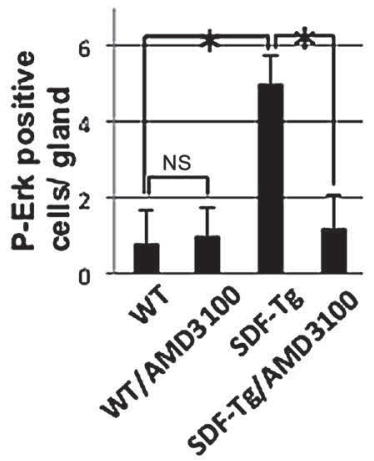

Corpus

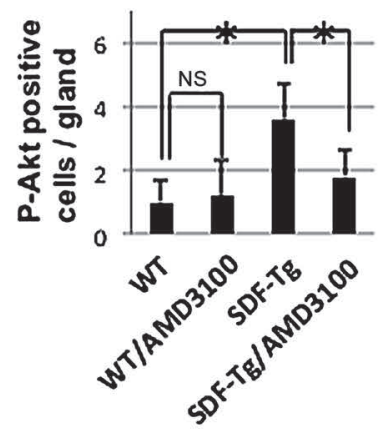

Figure 4 (A) Proliferation in WT and SDF-Tg mice stomachs stained with Ki67 ( $n=5$ per group). (B) WT and SDF-Tg mice with or without AMD3100 treatment stained for Ki67. The number of Ki67-positive cells per gland was determined by counting 10 glands per mouse ( $n=5$ per group, 3 -month-old mice). (C) WT and SDF-Tg mice treated with or without AMD3100 were stained with phospho-Akt or phospho-Erk ( $n=5$ per group). Data shown are mean \pm SE. ${ }^{*} p<0.05$. Scale bars $=100 \mu \mathrm{m}$.

in the stomach of SDF-Tg mice compared with WT mice (figure 5C). We also used immunohistochemistry to confirm the recruitment of Grem 1 (+) MSCs to the gastric mucosa of SDF$\mathrm{Tg}$ mice in combination with $H$ felis or IL-1 $\beta$ overexpression (figure 6A). Finally, we examined the direct chemotactic ability of SDF-1 on BM-MSCs in a migration assay using isolated BMMSCs stimulated with mouse gastric extracts from WT or SDF$\mathrm{Tg}$ mice in a Boyden chamber assay. Gastric protein extracts isolated from SDF-Tg mice induced a significant increase in cell migration of BM-MSCs compared with those isolated from WT mice, and this migration rate was reduced by the CXCR4 inhibitor AMD3100 (figure 6B). These findings clearly show that chemotaxis induced by the gastric microenvironment found in our SDF-Tg mice was mediated, at least in part, through the SDF1-CXCR4 pathway.

\section{DISCUSSION}

While SDF-1/CXCR4 signalling has been linked to numerous pathophysiological processes, the consequences of upregulated expression have not been well defined. In the current study we show that SDF-1 overexpression under the control of the H/KATPase $\beta$ promoter does not lead to severe inflammation but, nevertheless, is able to induce gastric dysplasia and tumour formation. Overexpression of SDF-1 directly stimulates the proliferation of gastric epithelial progenitor cells with increases in Ki67 and CXCR4-positive cells in the progenitor zone and an expansion of the K19-positive lineage. In addition, SDF-1 overexpression increased CXCR4-positive fibroblastic cells, especially aSMA-positive myofibroblasts, probably through recruitment of Grem1 + MSCs. Despite the limited effect on the induction of inflammation, SDF-1 overexpression appeared to synergise with either $H$ felis infection or IL-1 $\beta$ overexpression, resulting in severe inflammation with a marked increase of F4/80-positive macrophages. Overexpression of SDF-1 also appeared to synergise with proinflammatory cytokines through the activation of macrophages and promotion of macrophage survival. Taken together, our data offer direct evidence that elevation of a single CXC chemokine, SDF-1, promotes gastric carcinogenesis through both direct effects on gastric epithelial progenitors and through modulation of the gastric progenitor niche, consistent with prior clinical evidence that high levels of SDF-1 in patients are associated with gastric cancer (figure 7). ${ }^{37}$

SDF-1 has been thought to be the key chemokine responsible for the mobilisation and recruitment of inflammatory cells from the BM to sites of inflammation or cancer. ${ }^{17}$ SDF-1 is upregulated in a number of inflammatory diseases. ${ }^{18}$ Deletion of the CXCR4 receptor in specific haematopoietic subsets clearly impairs the recruitment of these cells, indicating that the SDF1-CXCR 4 axis is required for normal leucocyte trafficking. ${ }^{38}$ Our data, however, suggest that high levels of SDF-1 in peripheral 
A

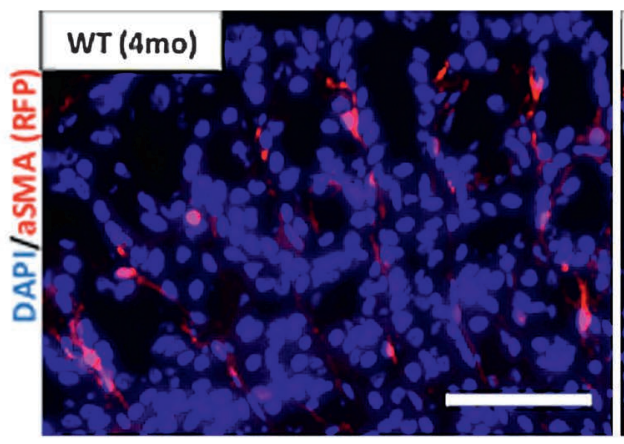

B

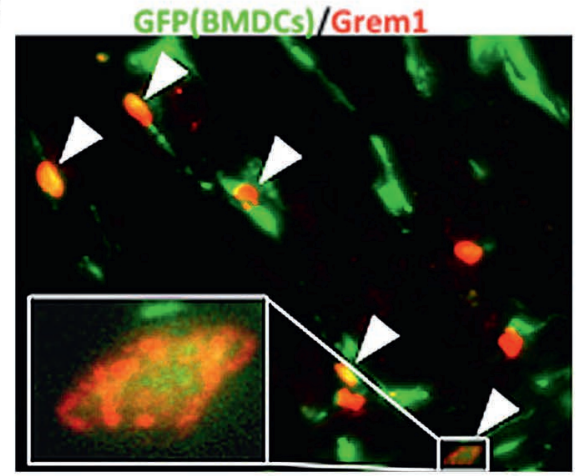

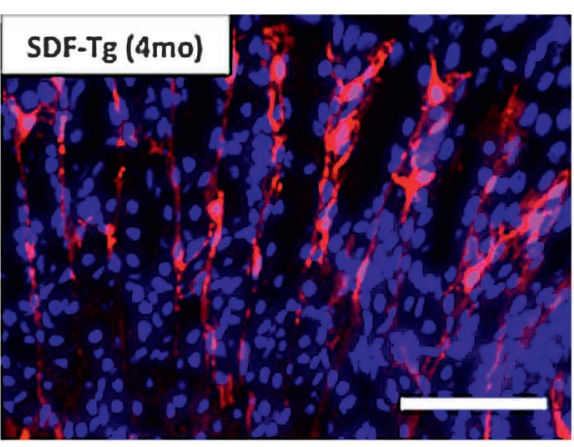

C

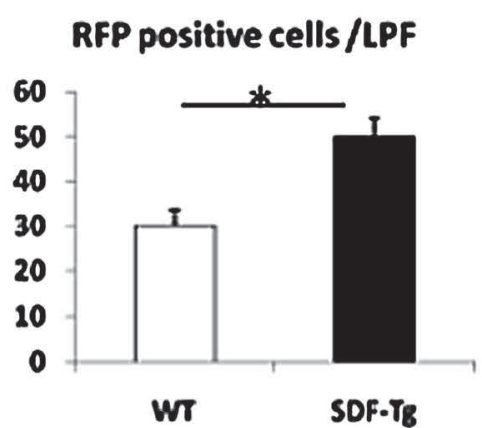

Figure 5 (A) $\alpha$-smooth muscle actin ( $\alpha$ SMA)-positive cells were expanded in SDF-Tg mice. Frozen sections from $\alpha$ SMA/RFP mice crossed with either WT or SDF-Tg mice were observed under a fluorescent microscope ( $n=3$ per group). Red: red fluorescent protein (RFP); blue: 4',6-diamidino-2 phenylindole (DAPI). Original magnification $\times 100$. (B) UBC-GFP traced BM-derived cells in the gastric stroma express Grem1 by immunostaining. Gastric tissue from SDF-Tg mice transplanted with UBC-GFP bone marrow was stained with anti-Gremlin 1 antibody. Green: bone marrow-derived cells; red: Gremlin 1. (C) mRNA expression of Gremlin 1 was increased in SDF-Tg mice compared with WT mice at 6 months ( $n=4$ per group). Results shown are mean + SE. ${ }^{*} \mathrm{p}<0.05$. Scale bars $=100 \mu \mathrm{m}$.

tissues such as the stomach are not sufficient for recruitment of leucocytes, since the SDF-1 Tg mice showed little inflammation at early time points. It was only at later time points that we observed a significant increase in F4/80-positive macrophages, and SDF-1 overexpression in combination of IL-1 $\beta$ overexpression resulted in a much more severe inflammatory response. It is likely that part of the explanation relates to the endogenous levels of BM SDF-1 which prevented the egress of BM cells. ${ }^{39}$ The key to the recruitment of inflammatory cells from BM may depend as much on the degradation of BM SDF-1 in response to proinflammatory cytokines such as IL- $1 \beta$ as it does on high levels of SDF-1 in peripheral tissue. ${ }^{25} 40$ In addition, our data would suggest that SDF-1 might contribute to inflammation by activating and stabilising macrophages, along with its effects on chemotaxis, consistent with previous reports. $^{41} 42$

Our data support the notion that one target of SDF-1 signalling is directly on gastric epithelial progenitors. We found CXCR4-positive cells in the isthmus of the normal gastric corpus and in the lower third of the normal antral glands. These portions of the gastric corpus and antrum contain gastric stem and progenitor cells, and also the Ki67-positive progenitor cells. In addition, in SDF-1 Tg mice we observed a gradual increase in CXCR4-EGFP-positive epithelial cells, consistent with an amplification of CXCR4-positive progenitors. Thus, our findings suggest that, in the gastric epithelium, SDF-1 secreted by gastric myofibroblasts regulates the proliferation and possibly the location of gastric epithelial progenitors. CXCR4 expression has been observed in the CNS and has been postulated to be important in regulating the migration of progenitor cells in postnatal brain. ${ }^{32}$ The downward migration of the proliferative zone commonly observed during gastric preneoplasia, for example, could in theory be related in part to SDF-1 expression by MSC-associated stromal cells which tend to expand at the base of the gastric glands. CXCR4 is also found in many cancer cell lines where it has been associated with tumour growth and metastasis, ${ }^{43}$ and the expression of CXCR4 by gastric progenitors could account for this finding of expression in cancer cells.

However, the most prominent effects of SDF-1 appeared to be the expansion of $\alpha \mathrm{SMA}$-positive myofibroblasts in the gastric mucosa. At early time points, much of this expansion appears to be due to proliferation of resident tissue mesenchymal cells, since we found only a small number of BM-derived aSMApositive myofibroblasts in our BM transplantation studies. Nevertheless, the data are consistent with the likely presence of MSCs in most peripheral tissues such as the stomach and the slow time course for BM-derived cell recruitment prior to the development of dysplasia. ${ }^{19}$ In previous studies we established Gremlin 1 as a putative marker for MSCs that give rise to myofibroblasts. ${ }^{19}$ Indeed, in the current study we found that Grem 1 mRNA expression was significantly upregulated in SDFTg mice when compared with WT mice, particularly in the setting of $H$ felis. We also showed that Grem1 + MSCs express CXCR4 and migrate in response to SDF-1 expression. In previous studies we also demonstrated that CXCR4 function was integral to the development of myofibroblasts as well, since TGF $\beta$ can induce myofibroblastic differentiation from MSCs through upregulation of SDF-1 production and is inhibited by 
A
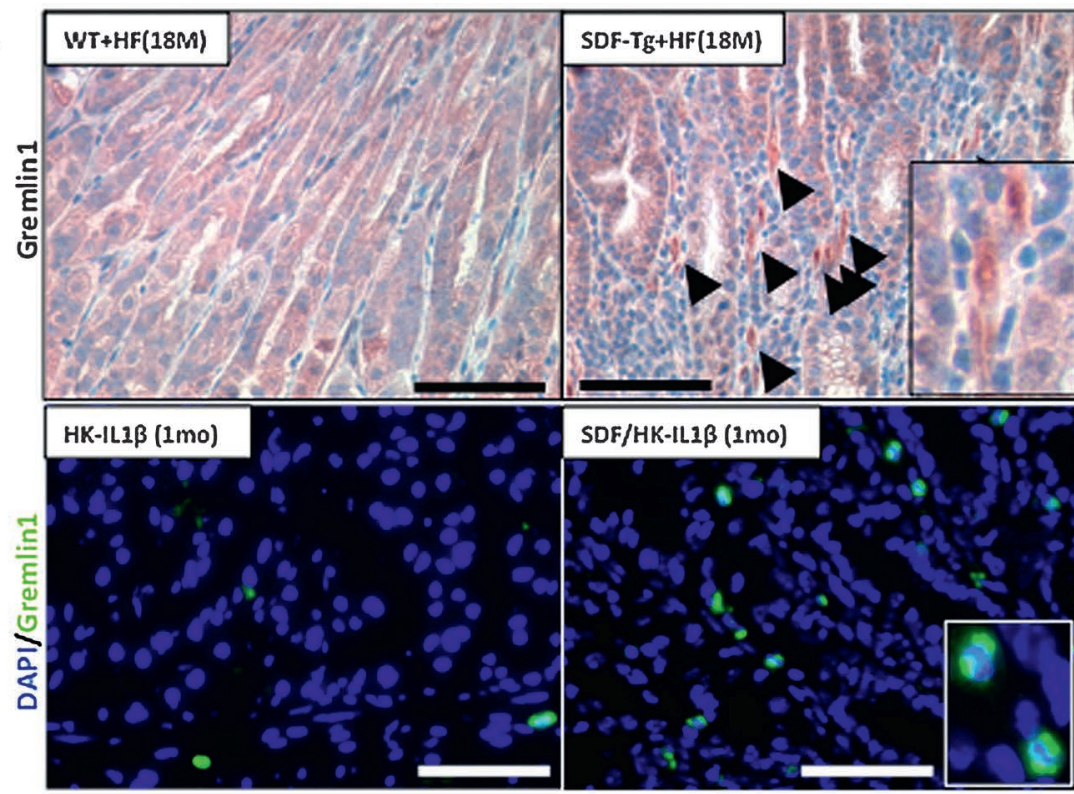

SDF/HK-ILIB (1mo)

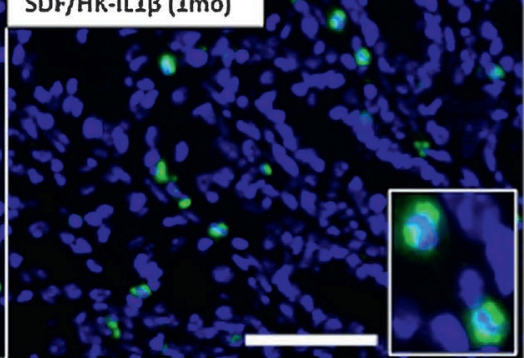

Gremlin1

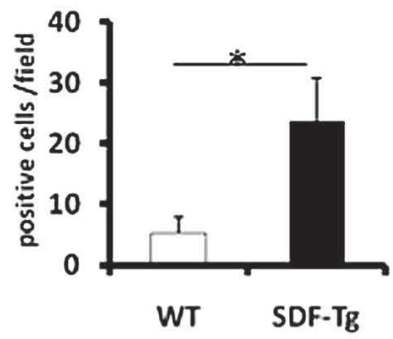

B Migrated cell area (\% of the field)

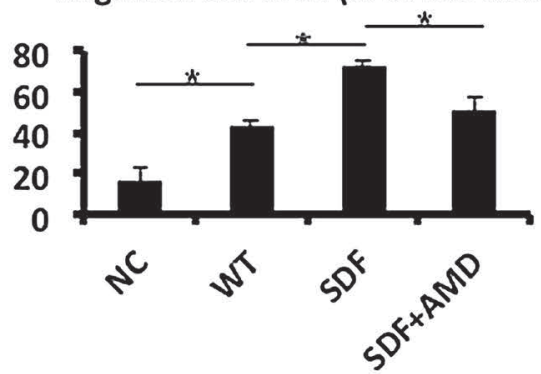

Figure 6 (A) Gremlin 1-positive cells were increased in SDF-Tg mice compared with WT mice infected with Helicobacter felis. Representative paraffin sections from stomachs are shown (top). Gremlin 1-positive cells were increased in SDF/H/K-ATPase-IL-1 $\beta$ double transgenic mice compared with H/K-ATPase-IL-1 $\beta$ mice (bottom). Frozen sections from SDF/H/K-ATPase-IL-1 $\beta$ or H/K-ATPase-IL-1 $\beta$ mice were stained ( $n=3$ per group). Green: Gremlin 1; blue: 4',6-diamidino-2-phenylindole (DAPI). Original magnification $\times 100$. (B) Transwell migration assay demonstrated the migration of bone marrow mesenchymal stem cells (BM-MSCs) in response to stromal cell-derived factor-1 (SDF-1) in 3-month-old mice. BM-MSCs were incubated with the gastric mixture from WT or SDF-Tg mice. SDF-1-induced BM-MSC migration was significantly inhibited by AMD3100. Results shown are mean $+\mathrm{SE} .{ }^{*} \mathrm{p}<0.05$ in each comparison indicated.

the CXCR4 antagonist AMD3100. ${ }^{19}$ While CXCR4 antagonism could also inhibit non-SDF-1 ligands such as ubiquitin, we believe that, in our SDF1-Tg driven model, the consequences of CXCR4 antagonism are primarily due to the loss of SDF1-related signalling.

In conclusion, the results presented here show that SDF-1/ CXCR4 signalling is important to the gastric epithelial niche. SDF-1 is normally produced by MSC-associated stromal cells, and increased SDF-1 promotes the proliferation of both epithelial cells and the expansion of stromal cells. While SDF-1 is a weak inflammatory chemokine, upregulation of SDF-1/CXCR4 pathway can synergise with other proinflammatory molecules (such as IL-1 $\beta$ ) and thus contribute to neoplasia. Taken together, the SDF/CXCR4 signalling pathway represents a promising target for future cancer prevention and treatment.
Figure 7 Schematic hypothesis of stromal cell-derived factor-1 (SDF-1)induced carcinogenesis. IL, interleukin; MSCs, mesenchymal stem cells; aSMA, $\alpha$-smooth muscle actin.

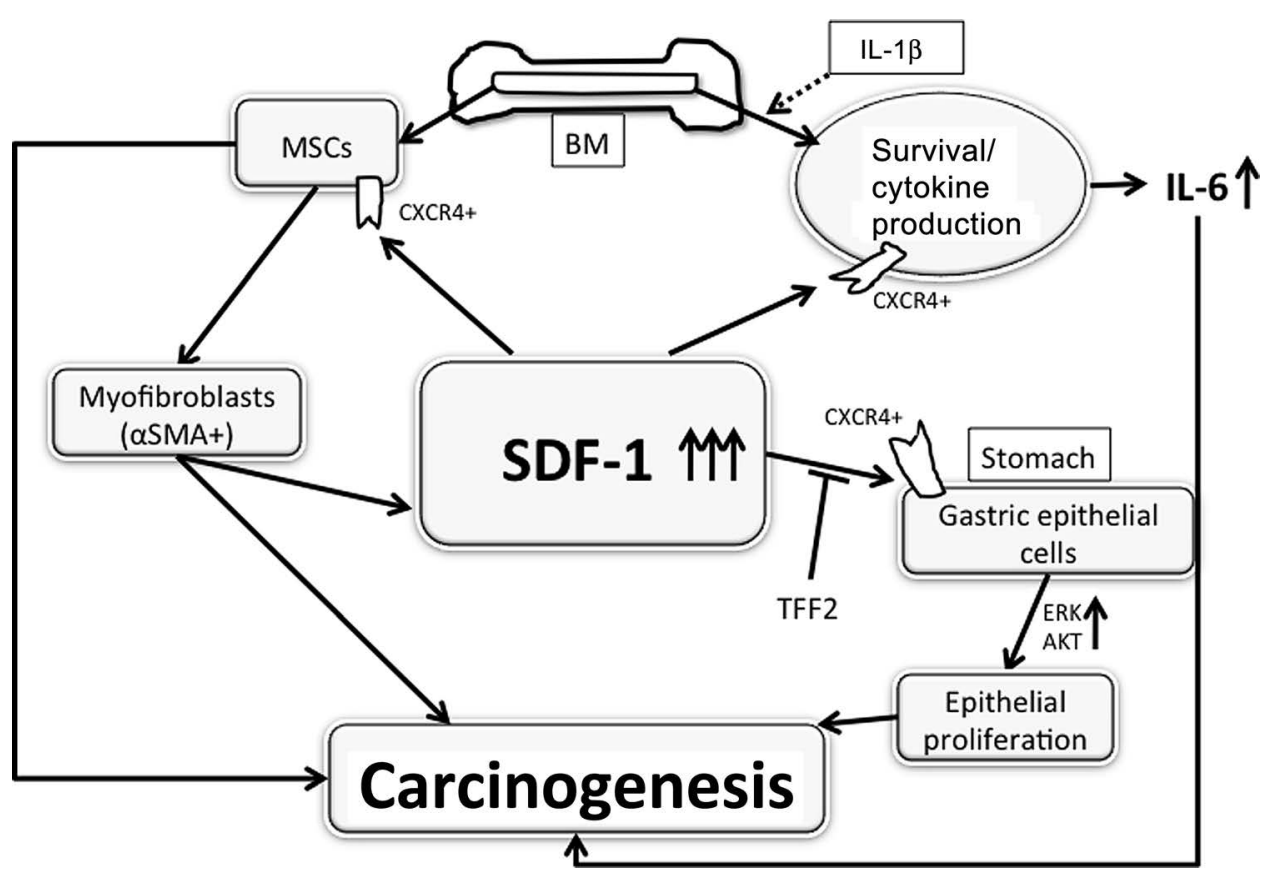


Acknowledgements The authors thank Kelly S Betz, Ashley Whelan, Justin DeGrazia and Chintan Kapadia for their help with animal studies.

Contributors Study concept and design, obtained funding and study supervision: TCW. Acquisition of data: WS, HA. Analysis and interpretation of data: WS, HA, CBW, DW, JF, SM, SA, ZD, MO, TCW. Drafting of manuscript: WS, HA, CBW, DW, MO, TCW.

Funding This research was supported by grants from the National Institute of Health grants 5R01CA093405-08 (TCW). WS was supported by the Japan Society for the Promotion of Science. MO is supported by the Deutsche Krebshilfe.

Competing interests None.

Provenance and peer review Not commissioned; externally peer reviewed.

\section{REFERENCES}

1. Fox JG, Wang TC. Inflammation, atrophy, and gastric cancer. J Clin Invest 2007;117:60-9.

2. Nickoloff BJ, Ben-Neriah Y, Pikarsky E. Inflammation and cancer: is the link as simple as we think? J Invest Dermatol 2005;124:x-xiv.

3. Grivennikov SI, Greten FR, Karin M. Immunity, inflammation, and cancer. Cell 2010;140:883-99.

4. Nagasawa T, Hirota S, Tachibana K, et al. Defects of B-cell lymphopoiesis and bone-marrow myelopoiesis in mice lacking the CXC chemokine PBSF/SDF-1. Nature 1996;382:635-8.

5. Lazarini F, Tham TN, Casanova $\mathrm{P}$, et al. Role of the alpha-chemokine stromal cellderived factor (SDF-1) in the developing and mature central nervous system. Glia 2003; 42:139-48.

6. Zou YR, Kottmann AH, Kuroda $M$, et al. Function of the chemokine receptor CXCR4 in haematopoiesis and in cerebellar development. Nature 1998;393:595-9.

7. Tachibana $\mathbf{K}$, Hirota S, lizasa $\mathrm{H}$, et al. The chemokine receptor CXCR4 is essential for vascularization of the gastrointestinal tract. Nature 1998;393:591-4.

8. De Clercq E. The AMD3100 story: the path to the discovery of a stem cell mobilizer (Mozobil). Biochem Pharmacol 2009;77:1655-64.

9. Eash KJ, Means JM, White DW, et al. CXCR4 is a key regulator of neutrophil release from the bone marrow under basal and stress granulopoiesis conditions. Blood 2009;113:4711-19.

10. Petit I, Jin D, Rafii S. The SDF-1-CXCR4 signaling pathway: a molecular hub modulating neo-angiogenesis. Trends Immunol 2007;28:299-307.

11. Duda DG, Kozin SV, Kirkpatrick ND, et al. CXCL12 (SDF1alpha)-CXCR4/CXCR7 pathway inhibition: an emerging sensitizer for anticancer therapies? Clin Cancer Res 2011;17:2074-80.

12. Hattori K, Heissig B, Tashiro K, et al. Plasma elevation of stromal cell-derived factor-1 induces mobilization of mature and immature hematopoietic progenitor and stem cells. Blood 2001:97:3354-60.

13. Hiasa K, Ishibashi M, Ohtani K, et al. Gene transfer of stromal cell-derived factor-1alpha enhances ischemic vasculogenesis and angiogenesis via vascular endothelial growth factor/endothelial nitric oxide synthase-related pathway: next-generation chemokine therapy for therapeutic neovascularization. Circulation 2004; 109:2454-61.

14. Yamaguchi J, Kusano KF, Masuo 0, et al. Stromal cell-derived factor-1 effects on ex vivo expanded endothelial progenitor cell recruitment for ischemic neovascularization. Circulation 2003;107:1322-8.

15. Karin N. The multiple faces of CXCL12 (SDF-1alpha) in the regulation of immunity during health and disease. J Leukoc Biol 2010;88:463-73.

16. Balkwill F. Cancer and the chemokine network. Nat Rev Cancer 2004;4:540-50.

17. Orimo A, Gupta PB, Sgroi DC, et al. Stromal fibroblasts present in invasive human breast carcinomas promote tumor growth and angiogenesis through elevated SDF-1/CXCL12 secretion. Cell 2005;121:335-48.

18. Tu S, Bhagat G, Cui G, et al. Overexpression of interleukin-1beta induces gastric inflammation and cancer and mobilizes myeloid-derived suppressor cells in mice. Cancer Cell 2008;14:408-19.
19. Quante M, Tu SP, Tomita H, et al. Bone marrow-derived myofibroblasts contribute to the mesenchymal stem cell niche and promote tumor growth. Cancer Cell 2011:19:257-72

20. Balkwill F. The significance of cancer cell expression of the chemokine receptor CXCR4. Semin Cancer Biol 2004;14:171-9.

21. Mishra PJ, Humeniuk R, Medina DJ, et al. Carcinoma-associated fibroblast-like differentiation of human mesenchymal stem cells. Cancer Res 2008;68:4331-9.

22. Spaeth EL, Dembinski JL, Sasser AK, et al. Mesenchymal stem cell transition to tumor-associated fibroblasts contributes to fibrovascular network expansion and tumor progression. PloS One 2009; 4:e4992.

23. Direkze NC, Hodivala-Dilke K, Jeffery $\mathrm{R}$, et al. Bone marrow contribution to tumor associated myofibroblasts and fibroblasts. Cancer Res 2004;64:8492-5.

24. Mishra P, Banerjee D, Ben-Baruch A. Chemokines at the crossroads of tumorfibroblast interactions that promote malignancy. J Leukoc Biol 2011;89:31-9.

25. Kerfoot SM, Andonegui G, Bonder CS, et al. Exogenous stromal cell-derived factor-1 induces modest leukocyte recruitment in vivo. Am J Physiol Heart Circ Physiol 2008:294:H2524-34.

26. Rogers AB, Houghton J. Helicobacter-based mouse models of digestive system carcinogenesis. Methods Mol Biol 2009;511:267-95.

27. El-Omar EM, Carrington M, Chow WH, et al. Interleukin-1 polymorphisms associated with increased risk of gastric cancer. Nature 2000;404:398-402.

28. Brembeck FH, Moffett J, Wang TC, et al. The keratin 19 promoter is potent for cellspecific targeting of genes in transgenic mice. Gastroenterology 2001;120:1720-8.

29. Quante M, Marrache F, Goldenring JR, et al. TFF2 mRNA transcript expression marks a gland progenitor cell of the gastric oxyntic mucosa. Gastroenterology 2010;139:2018-27.e2.

30. Lu DY, Tang CH, Yeh WL, et al. SDF-1alpha up-regulates interleukin-6 through CXCR4, PI3K/Akt, ERK, and NF-kappaB-dependent pathway in microglia. Eur $J$ Pharmacol 2009;613:146-54.

31. Dubeykovskaya Z, Dubeykovskiy A, Solal-Cohen J, et al. Secreted trefoil factor 2 activates the CXCR4 receptor in epithelial and lymphocytic cancer cell lines. J Biol Chem 2009;284:3650-62

32. Tran PB, Banisadr G, Ren D, et al. Chemokine receptor expression by neural progenitor cells in neurogenic regions of mouse brain. $J$ Comp Neuro 2007; 500:1007-33

33. Barker $\mathbf{N}$, Huch $\mathrm{M}$, Kujala $\mathrm{P}$, et al. Lgr5 $(+\mathrm{ve})$ stem cells drive self-renewal in the stomach and build long-lived gastric units in vitro. Cell Stem Cell 2010;6:25-36.

34. Brittan M, Wright NA. Gastrointestinal stem cells. J Pathol 2002;197:492-509.

35. Sneddon JB, Zhen HH, Montgomery K, et al. Bone morphogenetic protein antagonist gremlin 1 is widely expressed by cancer-associated stromal cells and can promote tumor cell proliferation. Proc Natl Acad Sci U S A 2006;103:14842-7.

36. Wynn RF, Hart CA, Corradi-Perini C, et al. A small proportion of mesenchymal stem cells strongly expresses functionally active CXCR4 receptor capable of promoting migration to bone marrow. Blood 2004;104:2643-5.

37. Iwasa S, Yanagawa T, Fan J, et al. Expression of CXCR4 and its ligand SDF-1 in intestinal-type gastric cancer is associated with lymph node and liver metastasis. Anticancer Res 2009;29:4751-8.

38. Sugiyama T, Kohara H, Noda M, et al. Maintenance of the hematopoietic stem cell pool by CXCL12-CXCR4 chemokine signaling in bone marrow stromal cell niches. Immunity 2006:25:977-88.

39. Cashen AF, Lazarus HM, Devine SM. Mobilizing stem cells from normal donors: is it possible to improve upon G-CSF? Bone Marrow Transplant 2007;39:577-88.

40. Broxmeyer HE, Orschell CM, Clapp DW, et al. Rapid mobilization of murine and human hematopoietic stem and progenitor cells with AMD3100, a CXCR4 antagonist. $J$ Exp Med 2005;201:1307-18.

41. Lee Y, Gotoh A, Kwon HJ, et al. Enhancement of intracellular signaling associated with hematopoietic progenitor cell survival in response to SDF-1/CXCL12 in synergy with other cytokines. Blood 2002;99:4307-17.

42. Hodohara K, Fujii N, Yamamoto N, et al. Stromal cell-derived factor-1 (SDF-1) acts together with thrombopoietin to enhance the development of megakaryocytic progenitor cells (CFU-MK). Blood 2000:95:769-75.

43. Yasumoto K, Koizumi K, Kawashima A, et al. Role of the CXCL12/CXCR4 axis in peritoneal carcinomatosis of gastric cancer. Cancer Res 2006:66:2181-7. 